\title{
Training various machine learning methods with advanced evolutionary optimization algorithms
}

\author{
Peter Poposki \\ Faculty of Computer Sciences, Saint Petersburg State University, Russia
}

\begin{abstract}
In the present research various machine learning methods are trained with advanced evolutionary optimization algorithms. Furthermore, a hybrid machine learning method is proposed as an optimized machine learning method based on random forest for modeling and simulation. The accuracy of the model has been improved via various evolutionary optimization algorithms. Several case studies and comparative analysis are discussed.
\end{abstract}

\section{References:}

Raat, W., Smeets, M., Henrard, S., Aertgeerts, B., Penders, J., Droogne, W., Mullens, W., Janssens, S., Vaes, B. Machine learning optimization of an electronic health record audit for heart failure in primary care (2022) ESC Heart Failure

Feng, Q., Maier, W., Stehle, T., Möhring, H.-C. Optimization of a clamping concept based on machine learning (2022) Production Engineering.

Mosavi, Amir. "The Large Scale System of Multiple Criteria Decision Making; Pre-processing." IFAC Proceedings Volumes 43.8 (2010): 354-359.

Fernández, M., Fritzen, F., Weeger, O. Material modeling for parametric, anisotropic finite strain hyperelasticity based on machine learning with application in optimization of metamaterials (2022) International Journal for Numerical Methods in Engineering.

Liu, J.-J., Liu, J.-C. Permeability Predictions for Tight Sandstone Reservoir Using Explainable Machine Learning and Particle Swarm Optimization (2022) Geofluids.

Kalbasi, Rasool, et al. "Finding the best station in Belgium to use residential-scale solar heating, one-year dynamic simulation with considering all system losses: economic analysis of using ETSW." Sustainable Energy Technologies and Assessments 45 (2021): 101097.

Venkata Rao, K., Parimi, S., Suvarna Raju, L., Suresh, G. Modelling and optimization of weld bead geometry in robotic gas metal arc-based additive manufacturing using machine learning, finite-element modelling and graph theory and matrix approach (2022) Soft Computing, .

Liu, Q., Zeng, Z., Jin, Y. Distributed machine learning, optimization and applications (2022) Neurocomputing.

Qasem, Sultan Noman, et al. "Estimating daily dew point temperature using machine learning algorithms." Water 11.3 (2019): 582.

Mosavi, A. (2010). Multiple criteria decision-making preprocessing using data mining tools. arXiv preprint arXiv:1004.3258.

Karballaeezadeh, N., Mohammadzadeh S, D., Shamshirband, S., Hajikhodaverdikhan, P., Mosavi, A., \& Chau, K. W. (2019). Prediction of remaining service life of pavement using an optimized support vector 
machine (case study of Semnan-Firuzkuh road). Engineering Applications of Computational Fluid Mechanics, 13(1), 188-198.

Khan, H., Wahab, F., Hussain, S., Khan, S., Rashid, M. Multi-object optimization of Navy-blue anodic oxidation via response surface models assisted with statistical and machine learning techniques (2022) Chemosphere.

Sui, Z., Sui, Y., Wu, W. Multi-objective optimization of a microchannel membrane-based absorber with inclined grooves based on CFD and machine learning (2022) Energy

Zhang, H., He, B., Lu, G., Zhu, Y. A simulation and machine learning based optimization method for integrated pedestrian facilities planning and staff assignment problem in the multi-mode rail transit transfer station (2022) Simulation Modelling Practice and Theory.

Mosavi, Amir, and Atieh Vaezipour. "Reactive search optimization; application to multiobjective optimization problems." Applied Mathematics 3.10A (2012): 1572-1582.

Mosavi, A., \& Rabczuk, T. (2017). Learning and intelligent optimization for material design innovation. In International Conference on Learning and Intelligent Optimization (pp. 358-363). Springer, Cham.

Mosavi, A., \& Rabczuk, T. (2017). Learning and intelligent optimization for material design innovation. In 11th International Conference on Learning and Intelligent Optimization, LION 2017 (pp. 358-363). Springer Verlag.

Samadianfard, Saeed, et al. "Wind speed prediction using a hybrid model of the multi-layer perceptron and whale optimization algorithm." Energy Reports 6 (2020): 1147-1159.

Shabani, Sevda, et al. "Modeling pan evaporation using Gaussian process regression K-nearest neighbors random forest and support vector machines; comparative analysis." Atmosphere 11.1 (2020): 66.

Dineva, Adrienn, et al. "Review of soft computing models in design and control of rotating electrical machines." Energies 12.6 (2019): 1049.

Torabi, Mehrnoosh, et al. "A hybrid machine learning approach for daily prediction of solar radiation." International Conference on Global Research and Education. Springer, Cham, 2018. 